\title{
Imagen corporal, depresión y autoestima: un estudio con preadolescentes
}

\author{
Laura Peris Reig'
}

\section{RESUMEN}

La adolescencia es la etapa en la que se configura el autoconcepto de manera más potente, siendo también cuando comienzan muchos de los trastornos de la conducta alimentaria [TCA] o comportamientos inadecuados con respecto a hábitos saludables. Son muchos los factores que influyen en el desarrollo de una mayor o menor insatisfacción con la imagen corporal, de entre los cuales destacan en esta etapa los factores biológicos o genéticos, los factores socioculturales, y los psicológicos. Tiene especial importancia la cultura en la que está inmerso el individúo, siendo en este caso una cultura donde la delgadez y el atractivo físico están íntimamente relacionados.

Con el fin de conocer cuándo comienzan a ser significativos estos factores, se ha estudiado la relación entre la insatisfacción con la imagen corporal y la autoestima en niños en etapa preadolescente [entre 10 y 12 años de edad]. Para ello, se han aplicado tres instrumentos: el testevaluación de la insatisfacción con la imagen corporal - IMAGEN (Solano y Cano, 2010), la prueba BIA (BodylmageAssessment) de Collins [1991) adaptada a niños, y el Inventario de Depresión Infantil [CDI] de Kovacs [1992). La muestra contaba con 46 participantes de nacionalidad española, y los resultados cumplen la hipótesis de que el género femenino tiene mayor insatisfacción corporal que el masculino, el cual avanza según la edad, pues cuanto más mayores [más cerca de la adolescencia] más preocupaciones parecen tener con respecto a su aspecto físico, aumentando el riesgo de padecer un futuro TCA. De esta manera, la insatisfacción con la imagen corporal y la autoestima estarían íntimamente relacionadas.

\section{PALABRAS CLAVE}

Imagen corporal, autoestima, trastornos de la alimentación [TCA], infancia, adolescencia.

$1 \quad$ Universidad Complutense de Madrid. lauperis@estumail.ucm.es 


\section{ABSTRACT}

Adolescence is the stage in which the self-concept becomes more powerful, being the stage configured to be also when they start many of the eating disorders [ED] or inadequate habits regarding healthy behaviors. There are many factors that influence the development of a more or less dissatisfaction with the body image, among which stand out at this stage biological or genetic factors, sociocultural influences and psychological factors. Of particular importance is the culture in which the individual is involved, being in this case a culture where thinness and physical attractiveness are closely related.

In order to know when these factors start to become significant, it has been studied the relationship between dissatisfaction with body image and self-esteem in children in pre-adolescent stage [10 to 12 years old]. To do this, it has been used three instruments: the test evaluation of dissatisfaction with body image - IMAGE [Solano y Cano, 2010], the BIA test [Body Image Assessment] of Collins [1991] adapted to children, and Inventory Childhood depression [CDI] of Kovacs (1992). The sample is 46 participants of Spanish nationality, whose results meet the hypothesis that the female gender has greater body dissatisfaction than men, which advances according to age, because the higher (closer to adolescence) more worries seem to have with regard to their physical appearance, increasing the risk of a future ED. Thus, dissatisfaction with body image and self-esteem would be closely related.

\section{KEYWORDS}

Body image, self-esteem, eating disorders [ED], childhood, adolescence. 


\section{INTRODUCCIÓN}

El tema principal de este estudio es la imagen corporal y la autoestima en los niños, siendo dos factores que están íntimamente relacionados, especialmente en etapas como la pubertad/ adolescencia, donde la apariencia física es una de las preocupaciones más potentes buscando encajar y ser aceptado en el grupo de iguales y en los patrones de belleza ideales para una cultura o sociedad concreta. El problema de ello es que una gran insatisfacción corporal y una baja autoestima pueden derivar en trastornos de la alimentación que comienzan a ser más frecuentes, en general, en la etapa adolescente.

A continuación, se presenta la investigación realizada con una muestra de 46 sujetos de edades comprendidas entre los 10 y los 12 años, donde se pueden ver los datos de los resultados obtenidos con respecto a tres instrumentos que miden la insatisfacción corporal y la autoestima, estableciendo correlaciones entre ambas variables y comparando los resultados obtenidos en los distintos grupos de género y edad/curso.

El objetivo principal de esta investigación es conocer la relación entre la imagen corporal y la autoestima, y ver en qué medida esta afecta a los niños en etapa pre-adolescente de la sociedad actual.

Los resultados esperados en este estudio parten de la hipótesis de que, en comparación con otras investigaciones y datos obtenidos, las chicas sufren una mayor presión por cumplir con los ideales de belleza, lo cual lleva a tener mayores preocupaciones sobre su aspecto físico. Como consecuencia, el género femenino presentaría una mayor insatisfacción corporal que el masculino. También, a medida que se acercan a la adolescencia, estas preocupaciones aumentan, por lo que los participantes de mayor edad tendrían más preocupaciones y más insatisfacción que los más pequeños.

El presente estudio parte del Trabajo Final del Máster de Psicopedagogía, presentado en la Facultad de Educación de la Universidad Complutense de Madrid en junio de 2016, bajo la tutela académica de la Dra. Irene Solbes Canales.

\section{DEFINIENDO LOS TÉRMINOS}

\subsection{Definición de imagen corporal}

Son varios los autores que intentan definir este concepto. En primer lugar, Le Boulch [1986], citado por Gallego del Castillo [2010), aporta una definición en la que la imagen o esquema corporal se concibe como el conocimiento inmediato y continuo que un sujeto tiene de su propio cuerpo, ya sea en reposo o en movimiento, teniendo en cuenta la relación con sus diferentes partes, así como con el espacio y los objetos que tiene a su alrededor.

Schilder [1978], por su parte, estudió el esquema corporal en relación con el cerebro en pacientes, estudiando la sociología de la imagen corporal y sus bases fisiológicas. Es quien propuso una de las definiciones más aceptadas por ser muy completa al reunir aspectos neurológicos y psicoanalíticos. Así pues, define la imagen corporal como la idea del cuerpo que un sujeto se forma en su mente, o sea, la apariencia física que le atribuye a su cuerpo [Gallego del Castillo, 2010). 
Una de las definiciones más globales que encontramos sobre el término es la que presenta Raich [2010] quien, adoptando la definición de Rosen [1992] considera que la imagen corporal se refiere a la manera en que uno percibe, siente, imagina y actúa con respecto a su cuerpo, conteniendo así tres aspectos o componentes distintivos: el perceptual, el subjetivo y el conductual.

De esta manera, explica, la representación mental que cada persona tiene de sí misma (de su cuerpo] se basa en aspectos como: las medidas corporales, los pensamientos, sentimientos y valoraciones de nuestro cuerpo [peso, complejos...] y las consecuencias de cómo me siento con respecto a esos complejos en términos conductuales [vestirse de determinada manera, evitar ropa pegada o bikinis, peinado, comparaciones....

\subsection{Definición de los trastornos de la alimentación}

Aunque los trastornos de la alimentación o de la conducta alimentaria [TCA] han existido siempre, su significado ha ido cambiando a lo largo del tiempo. Principalmente, puede decirse que los TCA se caracterizan por una alteración en las costumbres alimentarias de un individuo que suele mostrar conflictos psicosociales y se valora en función del logro de una constante delgadez [Gómez, 2013].

Una definición muy clara es la que aportan Portela de Santana, Da Costa, Mora yRaich [2012], según la cual los TCA son considerados como enfermedades psiquiátricas graves, que implican alteraciones de comportamiento, actitudes e ingestión, además de presentar una preocupación exagerada por el peso y la forma corporal. Afirman que son perjudiciales para la salud nutricional y que suelen ser muy difíciles de tratar, además de ir asociados a baja calidad de vida, alta comorbilidad psicosocial y mortalidad prematura.

Otra definición parecida es la aportada por Salaberria, Rodríguez y Cruz [2007], citados por Gómez [2013], según la cual los TCA se consideran alteraciones psicológicas que presentan graves anormalidades en el comportamiento alimentario, contando con que los más habituales son la Anorexia Nerviosa [AN] y la Bulimia Nerviosa [BN].

\section{RELACIÓN ENTRE IMAGEN CORPORAL Y TCA}

Brunch [1962] señala que una imagen corporal negativa puede conllevar consecuencias emocionales como baja autoestima y, en algunos casos, pueden acarrear trastornos alimentarios.

Pastor y Bonilla [2000] defienden que estos modelos normativos son los que, en la sociedad actual, hacen referencia a estereotipos de extrema delgadez y estándares de belleza que persiguen altos niveles de perfeccionismo y éxito, lo cual requiere una exigencia y una competición que provoca alteraciones de la imagen y de la alimentación, especialmente en mujeres [Limiñana, 2013).

Gardner [1996], citado por Espina, Ortego, Ochoa de Alda, Yenes y Alemán [2001], hace referenciaa dos de los componentes de la imagen corporal que ya se han visto anteriormente: la percepción [tamaño, apariencia...] y la actitud [sentimientos y actitudes hacia el cuerpo]. Para él, el trastorno de la imagen corporal se basa en una preocupación exagerada por algún defecto [imaginario o exagerado] de la apariencia física, tal y como hemos visto en apartados anteriores. 
La relación que plantea él entre esta preocupación y los TCA es que la persona comienza a preocuparse en exceso por la opinión de los demás y a quitarse valor poniendo el enfoque solamente en el aspecto físico, de forma que estas personas llevarán a cabo conductas como oculta su cuerpo, realizar dietas en su alimentación y a centrarse en el ejercicio físico, llegando incluso a evitar las relaciones sociales.

\section{INVESTIGACIÓN}

\subsection{Objetivos}

El objetivo principal de este estudio era analizar la imagen corporal de un grupo de preadolescentes escolarizados en los últimos años de la etapa de educación Primaria, así como la relación entre esta variable y su autoestima, incluyendo la posible aparición de aspectos depresivos (tristeza, preocupaciones, disforia...].

Se ha escogido esta edad para comprobar si hay indicios antes de la adolescencia que apunten a la insatisfacción corporal que sufren muchos adolescentes debido a la presión social que se ejerce sobre el cuerpo humano y los estereotipos de belleza.

En vista de los resultados de anteriores investigaciones, se plantea la hipótesis de que, a mayor edad, aparecerá mayor insatisfacción corporal, dándose más casos en las mujeres que en los varones. Se espera además que haya una relación positiva entre la insatisfacción corporal y presentar una baja autoestima.

\subsection{Metogología}

\subsubsection{La muestra: participantes}

Los análisis contenidos en esta sección se han llevado a cabo sobre una muestra reducida e incidental, procedente de un centro escolar privado de la Comunidad de Madrid, ubicado en una zona socioeconómica media-alta de la ciudad, de donde proceden la mayor parte de sus alumnos. Interesa saber que los alumnos y familias que acuden al colegio son, en su gran mayoría, de nacionalidad y cultura española. Además, este centro obtiene en el CDI de la Comunidad Autónoma de Madrid calificaciones académicas más altas que la media de esta Comunidad en la mayoría de las áreas o materias.

A la hora de escoger la muestra, y debido a que el interés de la investigación giraba en torno a la prevención y detección precoz de la sintomatología abarcada en el marco teórico, se han seleccionado los grupos de 5ํ y 6ํㅜ de Educación Primaria. Por limitaciones de disponibilidad, la muestra consta de un total de 46 niños/as de entre 10 y 12 años, con una edad media de edad de 10'65 años. De estos participantes, 20 son de sexo masculino [12 alumnos en 50 curso y 9 alumnos en $6^{\circ}$ ], y 26 de sexo femenino [ 11 alumnas en $5^{\circ}$ curso y 14 alumnas en $6^{ }$).

En todo momento, tanto el procedimiento de participación de los sujetos como la evaluación de los mismos han sido supervisados por el equipo de psicólogos del centro, recogiéndose los datos en presencia de los maestros. Sólo se aplicaron los cuestionarios a los niños y niñas cuyos 
padres dieron su consentimiento previamente, siendo informados por la dirección del centro con antelación suficiente antes de la aplicación.

\subsubsection{Recursos utilizados [instrumentos]}

Para conocer la percepción y la satisfacción con la propia imagen corporal se les aplicó a los alumnos el cuestionario IMAGEN, seguido del BIA y del cuestionario de detección de depresión infantil CDI.

\subsubsection{Proceso de evaluación y obtención de resultados}

Debido a las características de la investigación, el análisis de los datos obtenidos para la obtención de resultados y su evaluación se ha realizado con el programa estadístico informático SPSS versión 16, habiendo aplicado las siguientes pruebas del programa estadístico: análisis descriptivos, análisis de frecuencia, prueba T de Student, Prueba Chi cuadrado [X2]

\subsection{Resultados}

Para describir las tendencias generales de respuesta que se han obtenido, se va a considerar inicialmente a todos los participantes de la muestra en su conjunto. Para ello, se ha realizado inicialmente unos análisis descriptivos generales, que quedan resumidos en las tablas presentes en los distintos apartados.

\section{Resultados generales}

Atendiendo a la clasificación de los sujetos en categorías de riesgo de TCA, los datos indican que la mitad de los participantes estarían ubicados en la categoría "ausencia-mínima" de insatisfacción corporal, mientras que un 43,5\% lo estaría en la categoría "moderada", y el 6,5\% restante en la categoría "marcada", no existiendo ningún participante que pueda ser incluido en la categoría de insatisfacción "severa", tal y como se muestra en el siguiente gráfico.

En cuanto al BIA, los participantes en general se auto identificaron con las figuras de complexión media, es decir, rodearon aquellas situadas en torno a la figura 3 y la 4 en una escala del 1 al 7 $[\mathrm{M}=3,85]$.

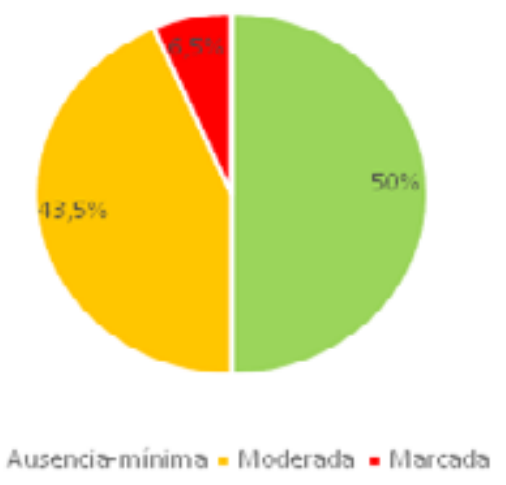

GRÁFICO 1. PORCENTAJES TOTALES DE INSATISFACCIÓN [IMAGEN] 
En cuanto a las figuras de identificación ideal, los sujetos seleccionaron aquellas situadas de nuevo en el centro, siendo pocos los sujetos que marcaron una figura distinta a la marcada en la tarea de auto identificación $(M=3,74)$.De hecho, tal y como se explicaba en apartados anteriores, al calcular la discrepancia entre la figura de auto identificación y la de identificación ideal, la puntuación obtenida es de $M=0,1087$, siendo 0 el valor que más cercano a la satisfacción corporal.

Por último, la puntuación general obtenida en el cuestionario CDI es de casi 20 en cuanto a su percentil $(M=19,57)$, lo cual se clasificaría como "Sin sintomatología" de entre las categorías siguientes: sin sintomatología, leve, severa, considerando los baremos correspondientes para el sexo masculino y para el femenino. Además, la media obtenida en este test para medir la autoestima es de 23,89, y la puntuación media en disforia alcanza un valor de 19,57, ambos categorizados como "Sin sintomatología".

Para obtener la frecuencia de síntomas depresivos en estos participantes, se han establecido como alternativa de la autora de la presente investigación 3 categorías que no siguen exactamente las marcadas por el baremo del manual del CDI, ya que la mayoría de niños/as se incluiría dentro de la categoría "sin sintomatología"propuesta en el cuestionario. Sin embargo, hay algunos sujetos cuya puntuación convertida a percentil eraalgo más alta [rozando el punto de corte para la categoría de "leve"]. Por ello, se ha establecido que, para un análisis de datos que muestre mejor la realidad de esta muestra, 0 equivaldría a puntuaciones bajas en cuanto a los percentiles, 1 a puntuaciones medias,aunque entrarían dentro de la categoría "sin sintomatología", y 2 a puntuaciones correspondientes con la categoría "leve".

Considerando esta nueva categorización, que permite afinar un poco más los resultados aun considerando que los baremos reales indican que no hay patología depresiva, es interesante conocer que el 91,3\% del grupo total se incluye en la categoría de depresión total denominada como "sin sintomatología", el 6,5\% se incluye en la categoría "casi leve" (equivalente al 1, no se consideraría indicativo de depresión según el manual], y el २,२\% entraría dentro de la categoría "leve". A continuación,el gráfico 2 representa estos resultados.

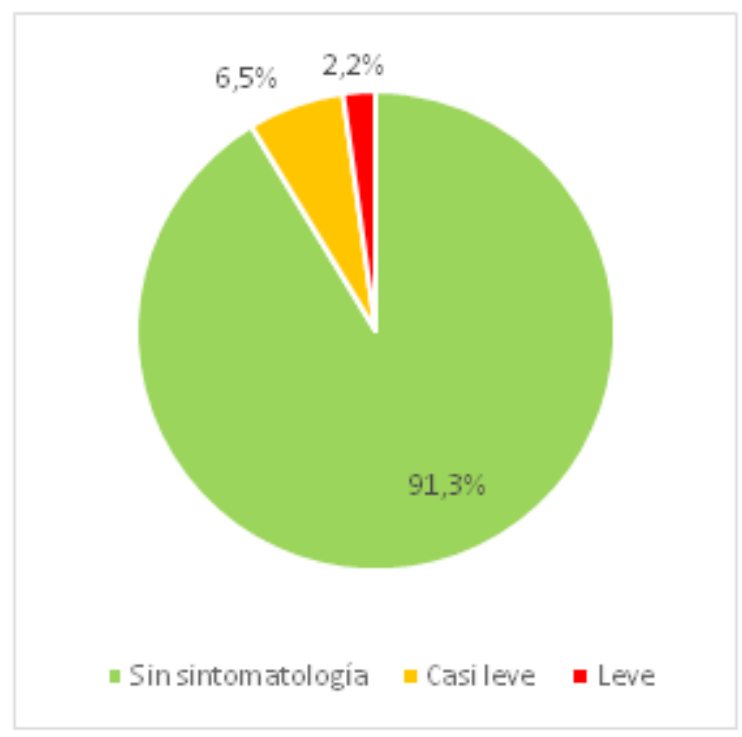

GRÁFICO 2: PORCENTAJES DE SINTOMATOLOGÍA DEPRESIVA, SEGÚN LA CATEGORIZACIÓN "ALTERNATIVA" PROPUESTA POR LA AUTORA DEL TRABAJO 


\section{Diferencias de género}

Para analizar la posible existencia de diferencias de género en las distintas puntuaciones cuantitativas continuas incluidas en el estudio (la puntuación total obtenida en la prueba de imagen corporal, test de las figuras, autoestima y depresión] se ha aplicado la prueba T de Student a las variables correspondientes.

Para organizar los resultados obtenidos, se presentan primero aquellos que han resultado ser estadísticamente significativos, teniendo en cuenta el pequeño tamaño de la muesta.

En este caso, podemos comprobar que la única diferencia notable se daen el test IMAGEN, concretamente entre la insatisfacción corporal que presentan los sujetos del sexo masculino $[M=8,4286]$, y la insatisfacción que tiene con respecto a su cuerpo el sexo femenino $[M=13,8400]$, siendo mayor en las chicas que en los chicos. Así pues, la prueba $T$ indica que sí hay diferencias entre ambos géneros en cuanto a la insatisfacción corporal $[\mathrm{t}[44]=-2,111 ; p=0.041]$.

Sin embargo, si nos fijamos en la puntuación obtenida en el BIA, la puntuación media de insatisfacción es más alta en el género femenino $[M=, 1600)$ que en el masculino $[M=, 0476]$, pero la prueba $T$ indica que no habría diferencias significativas entre ambos géneros en cuanto a la insatisfacción obtenida a través de la prueba $\mathrm{BIA}[\mathrm{t}[44]=-0,784 ; \mathrm{p}=0,437]$.

Por otro lado, llama la atención que en el cuestionario CDI de depresión infantil existen puntuaciones más altas para el género masculino que para el femenino, tanto en disforia $[M=35,00 ; M=26,00$; respectivamente] como en autoestima $(M=28,00 ; M=20,44]$ y en la puntuación de depresión total $(M=24,62 ; M=15,32]$.No obstante, de nuevo ninguna de estas diferencias de género resultó ser estadísticamente significativas.

A continuación, se presentan en la tabla 2 las diferencias en cuanto al género [21 chicos y 25 chicas] recogidas en la siguiente tabla, resumiendolos datos que presentan algunas diferencias y resaltado aquellas que son estadísticamente significativas.

\begin{tabular}{|l|c|c|c|}
\hline & Sexo & Media & Desviación típ. \\
\hline \multirow{2}{*}{$\begin{array}{l}\text { IMAGEN: Puntuación directa } \\
\text { insatisfacción * }\end{array}$} & Masculino & 8,429 & 6,853 \\
\cline { 2 - 4 } & Femenino & 13,84 & 9,919 \\
\hline \multirow{2}{*}{ BIA: Insatisfacción } & Masculino & 0,048 & 0,384 \\
\cline { 2 - 4 } & Femenino & 0,16 & 0,554 \\
\hline \multirow{2}{*}{ CDI: Disforia } & Masculino & 35 & 27,659 \\
\cline { 2 - 4 } & Femenino & 26 & 17,559 \\
\hline \multirow{2}{*}{ CDI: Autoestima } & Masculino & 28 & 27,342 \\
\cline { 2 - 4 } & Femenino & 20,44 & 19,462 \\
\hline \multirow{2}{*}{ CDI: Depresión total } & Masculino & 24,62 & 29,094 \\
\cline { 2 - 4 } & Femenino & 15,32 & 17,406 \\
\hline
\end{tabular}

TABLA 2: PRUEBA T - DIFERENCIAS GÉNERO

$$
\text { * } p<.05
$$




\section{Diferencias de curso}

Para conocer las diferencias que existen entre los distintos niveles educativos analizados [ 5 ํㅡ y $6^{0}$ de Educación Primaria] en cuanto a la insatisfacción corporal y a la autoestima y depresión entre los participantes de la muestra escogida, se ha aplicado la prueba T de Student, que, como se ha visto anteriormente, permite conocer si hay diferencias significativas entre ambos grupos. De la misma manera para organizar los resultados obtenidos, se presentan primero aquellos que han resultado ser estadísticamente significativos.

En este caso, podemos observar en la prueba IMAGEN que hay una diferencia significativa entre la insatisfacción corporal que presentan los sujetos de $5^{\circ}$ curso $[M=8,2609]$, y la insatisfacción corporal de los alumnos de $\mathrm{G}^{\mathrm{o}}$ curso $(\mathrm{M}=14,4783$ ], siendo mayor en los alumnos mayores, es decir los del curso superior, que en los pequeños. Esta relación es estadísticamente significativa según la prueba T de Student que se ha aplicado $[\mathrm{t}[44]=-2,476 ; p=0,017]$.

También aparece como significativa la diferencia entre los dos grupos de edad en la variable de Disforia del cuestionario CDI [t[44] = -2,030; $p=0,048]$, siendo mayor en los participantes de 6o curso que en los de 5o curso.

Sin embargo, si nos fijamos en la puntuación obtenida en el BIA, la puntuación media de insatisfacción es ligeramente más alta en los alumnos de 6o curso $[M=, 1304]$ que en los de 5o $(M=, 0870)$, aunque de nuevo en este caso la prueba $T$ indica que estas diferencias no son estadísticamente significativas $[\mathrm{t}[44]=-0,303 ; p=0,763]$.

Por último, en el cuestionario CDI de depresión infantil los alumnos del curso superior también presentan puntuaciones más altas de Depresión total $[M=23,26]$ con respecto a los del curso inferior $[M=15,87]$.Aunque, al igual que ocurría con el género, la prueba $T$ nos indica que no son diferencias estadísticamente significativas para las variables Autoestima $[\mathrm{t}[44]=-0,068 ; \mathrm{p}=$ 0,946] y Depresión Total [t[44] = -1,061; p =0,295].

\begin{tabular}{|l|c|c|c|}
\hline & Curso & Media & Desviación típ. \\
\hline \multirow{2}{*}{$\begin{array}{l}\text { IMAGEN: Puntuación directa } \\
\text { insatisfacción * }\end{array}$} & 5 & 8,261 & 6,870 \\
\hline \multirow{3}{*}{ BIA: Insatisfacción } & 6 & 14,478 & 9,890 \\
\hline \multirow{3}{*}{ CDI: Disforia * } & 5 & 0,087 & 0,417 \\
\cline { 2 - 4 } & 6 & 0,130 & 0,548 \\
\hline \multirow{3}{*}{ CDI: Autoestima } & 5 & 23,48 & 19,565 \\
\cline { 2 - 4 } & 5 & 36,74 & 24,477 \\
\hline \multirow{2}{*}{ CDI: Depresión total } & 6 & 24,13 & 21,302 \\
\cline { 2 - 4 } & 5 & 23,65 & 25,857 \\
\cline { 2 - 4 } & 6 & 15,87 & 21,24 \\
\hline
\end{tabular}

TABLA 3: PRUEBA T - DIFERENCIAS CURSO

$$
* p<.05
$$




\section{Correlaciones}

Para finalizar, nos propusimos conocer si había alguna relación entre las distintas variables consideradas en el estudio.

Con el fin de conocer las correlaciones totales [para toda la muestra] se han aplicado pruebas de correlaciones bivariadas, incluyendo todas las variables continuas como la edad, las puntuaciones obtenidas en el test IMAGEN, las puntuaciones del BIA y los resultados del CDI. Así pues, se ha optado por utilizar el Coeficiente de Correlación de Spearman, encontrándose los resultados obtenidos en la Tabla 4.

\begin{tabular}{|l|c|c|c|c|c|c|c|c|}
\hline & Edad & IMAGEN & $\begin{array}{c}\text { BIA: } \\
\text { Fig. que soy }\end{array}$ & $\begin{array}{c}\text { BIA: } \\
\text { Fig. ideal }\end{array}$ & $\begin{array}{c}\text { BIA: } \\
\text { Insatisfación }\end{array}$ & $\begin{array}{c}\text { CDI: } \\
\text { Disforia }\end{array}$ & $\begin{array}{c}\text { CDI: } \\
\text { Autoestima }\end{array}$ & $\begin{array}{c}\text { CDI: } \\
\text { Depresión total }\end{array}$ \\
\hline Edad & 1 & 0,215 & 0,085 & 0,17 & 0,003 &, $328^{*}$ & $-0,148$ & 0,058 \\
\hline IMAGEN & 0,215 & 1 &, $460^{* *}$ & 0,245 &, $508^{* *}$ &, $414^{* *}$ & 0,123 &, $306^{*}$ \\
\hline BIA: Fig. que soy & 0,085 &, $460^{* *}$ & 1 &, $792^{\star *}$ &, $695^{\star *}$ & 0,084 & $-0,046$ & 0,074 \\
\hline BIA: Fig. ideal & 0,17 & 0,245 &, $792^{* *}$ & 1 & 0,141 & $-0,003$ & $-0,052$ & 0,002 \\
\hline BIA: Insatisfacción & 0,003 &, $508^{* *}$ &, $695^{* *}$ & 0,141 & 1 & 0,222 & 0,008 & 0,165 \\
\hline CDI: Disforia &, $328^{*}$ &, $414^{* *}$ & 0,084 & $-0,003$ & 0,222 & 1 &, $537^{* *}$ &, $759^{* *}$ \\
\hline CDI: Autoestima & $-0,148$ & 0,123 & $-0,046$ & $-0,052$ & 0,008 &, $537^{* *}$ & 1 &, $896^{* *}$ \\
\hline CDI: Depresión total & 0,058 &, $306^{*}$ & 0,074 & 0,002 & 0,165 &, $759^{* *}$ &, $896^{* *}$ & 1 \\
\hline
\end{tabular}

TABLA 4. CORRELACIONES ENTRE VARIABLES

$$
\text { * } p<.05 ; * * p<.01
$$

En este caso, vemos que la percepción de la imagen corporal según el test IMAGEN se relaciona con niveles de insatisfacción en el BIA [ $r=.508, p<0.01]$ y, puesto que la correlación es positiva,podemos decir que cuanta más insatisfacción observamos a través del test IMAGEN, mayor insatisfacción se ha encontrado en el test BIA de las figuras de Collins [1991], lo cual tiene sentido pues ambos instrumentos miden constructos parecidos.

Así pues, podemos observar una correlación significativa entre la "Figura que soy" del BIA y la insatisfacción corporal del IMAGEN $[r=.460, p<0.01$ ]: cuanto mayor complexión física tiene la figura con la que se identifican [más corpulentos se ven], mayor es el riesgo de padecer un TCA, según el test IMAGEN.

Por otro lado, cuanto peor puntúan en el test IMAGEN (mayor insatisfacción corporal tienen], presentan niveles más altos de Disforia en el cuestionario de depresión CDI, siendo de hecho esta una relación bastante potente $[r=.414, p<0.05]$ y presentando también una correlación con los valores de Depresión Total de dicho cuestionario $[r=.306, p<0.05$ ). Parece por lo tanto que la insatisfacción corporal se relaciona con la depresión en general, y la disforia en particular.

Respecto a la inclusión dela edad como variable continua en el análisis de correlaciones, solo se ha observado una relación significativa entre esta variable y la Disforia medida a través del cuestionario CDI, observando así que los alumnos más mayores presentan mayor tendencia a presentar disforia; es decir, con la edad, aumenta la cantidad de pensamientos depresivos en los alumnos $(r=.328, p<0.05]$. 


\section{Correlaciones entre variables en los grupos de niños/niñas}

Además del análisis de correlaciones entre variables considerando a la muestra de participantes globalmente, se ha realizado un análisis posterior complementario segmentando la misma en dos grupos de niños/as, con el objetivo de ver si las correlaciones variaban en los dos subgrupos. Estas pruebas incluyen todas las variables continuas anteriores [edad y puntuaciones obtenidas en el test IMAGEN, BIA y CDI], utilizándose como en el caso anterior el Coeficiente de Correlación de Spearman.

En este caso, 21 de los participantes de la muestra total son de género masculino, independientemente de su edad o curso.

\begin{tabular}{|l|c|c|c|c|c|c|c|c|}
\hline & Edad & IMAGEN & $\begin{array}{c}\text { BIA: } \\
\text { Fig. que soy }\end{array}$ & $\begin{array}{c}\text { BIA: } \\
\text { Fig. ideal }\end{array}$ & $\begin{array}{c}\text { BIA: } \\
\text { Insatisfacción }\end{array}$ & $\begin{array}{c}\text { CDI: } \\
\text { Disforia }\end{array}$ & $\begin{array}{c}\text { CDI: } \\
\text { Autoestima }\end{array}$ & $\begin{array}{c}\text { CDI: } \\
\text { Depresión total }\end{array}$ \\
\hline Edad & 1 & 0,081 & 0,098 & 0,092 & 0,127 & 0,268 & $-0,211$ & 0,07 \\
\hline IMAGEN & 0,081 & 1 & 0,343 & 0,155 &, $479^{*}$ &, $480^{*}$ & 0,039 & 0,238 \\
\hline BIA: Fig. que soy & 0,098 & 0,343 & 1 &, $861^{* *}$ &, $584^{* *}$ & 0,21 & 0,21 & 0,28 \\
\hline BIA: Fig. ideal & 0,092 & 0,155 &, $861^{* *}$ & 1 & 0,106 & 0,249 & 0,327 & 0,375 \\
\hline BIA: Insatisfacción & 0,127 &, $479^{*}$ &, $584^{* *}$ & 0,106 & 1 & 0,131 & $-0,08$ & 0,031 \\
\hline CDI: Disforia & 0,268 &, $480^{*}$ & 0,21 & 0,249 & 0,131 & 1 &, $608^{* *}$ &, $803^{* *}$ \\
\hline CDI: Autoestima & $-0,211$ & 0,039 & 0,21 & 0,327 & $-0,08$ &, $608^{* *}$ & 1 &, $904^{* *}$ \\
\hline CDI: Depresión total & 0,07 & 0,238 & 0,28 & 0,375 & 0,031 &, $803^{* *}$ &, $904^{* *}$ & 1 \\
\hline
\end{tabular}

TABLA 5. CORRELACIONES ENTRE VARIABLES PARA LOS NIÑOS [VARONES]

$$
\text { * } p<.05 ; * \star p<.01
$$

Como podemos ver, no hay relaciones significativas entre la edad de los participantes y el resto de las variables en el caso de los niños. Sin embargo, se puede apreciar que la percepción de la imagen corporal según el test IMAGEN se relaciona con niveles de insatisfacción en el BIA [ $r=$ .479, $p$ < 0.05], tal y como ocurría en la muestra general. Esto indica quea más insatisfacción corporalen el test IMAGEN, más insatisfacción con la figura corporal en el test BIA presentan.

Por otro lado, vemos que los resultados del test IMAGEN [insatisfacción corporal) están relacionados en los varones con niveles altos de disforia, tal y como se puede observar en los datos obtenidos del cuestionario de depresión infantil CDI [ $r=.480, p<0.05$ ]. Este dato también concuerda con los resultados observados en la muestra global.

\begin{tabular}{|c|c|c|c|c|c|c|c|c|}
\hline & Edad & IMAGEN & $\begin{array}{c}\text { BIA: } \\
\text { Fig. que soy }\end{array}$ & $\begin{array}{l}\text { BIA: } \\
\text { Fig. ideal }\end{array}$ & $\begin{array}{c}\text { BIA: } \\
\text { Insatisfacción }\end{array}$ & $\begin{array}{c}\text { CDI: } \\
\text { Disforia }\end{array}$ & $\begin{array}{c}\text { CDI: } \\
\text { Autoestima }\end{array}$ & $\begin{array}{c}\text { CDl: } \\
\text { Depresión total }\end{array}$ \\
\hline Edad & 1 & 0,265 & $-0,022$ & 0,164 & $-0,099$ &, $432^{\star}$ & $-0,076$ & 0,107 \\
\hline IMAGEN & 0,265 & 1 &, $404^{*}$ & 0,181 &, $487^{\star}$ &, $505^{*}$ & 0,286 &, $491^{*}$ \\
\hline BIA: Fig. que soy & $-0,022$ &, $404^{\star}$ & 1 & ,711** & ,729** & 0,044 & $-0,238$ & $-0,12$ \\
\hline BIA: Fig. ideal & 0,164 & 0,181 &, $711^{* *}$ & 1 & 0,091 & $-0,147$ & $-0,387$ & $-0,378$ \\
\hline BIA: Insatisfacción & $-0,099$ &, $487^{*}$ & ,729** & 0,091 & 1 & 0,304 & 0,079 & 0,264 \\
\hline CDI: Disforia &, $432^{*}$ &, $505^{\star}$ & 0,044 & $-0,147$ & 0,304 & 1 &, $464^{*}$ & ,732 \\
\hline CDI: Autoestima & $-0,076$ & 0,286 & $-0,238$ & $-0,387$ & 0,079 &, $464^{*}$ & 1 &, $865^{\star \star}$ \\
\hline $\begin{array}{l}\text { CDI: Depresión } \\
\text { total }\end{array}$ & 0,107 &, $491^{*}$ & $-0,12$ & $-0,378$ & 0,264 & ,732** &, $865^{* *}$ & 1 \\
\hline
\end{tabular}

TABLA 6. CORRELACIONES ENTRE VARIABLES PARA LAS NIÑAS

$$
\text { * } p<.05 ; * \star p<.01
$$


En este caso, se observa que sí existe una relación entre la edad de las chicas participantes y la Disforia, pues según el curso y la edad en la que se encuentran las participantes, varía. En este caso, según el cuestionario CDI, va aumentando la Disforia entre las chicas conforme aumenta su edad $[r=.432, p<0.05]$.

Además, se puede constatar la existencia también de una relación entre las puntuaciones de insatisfacción corporal obtenidas a través del test IMAGEN y las puntuaciones de depresión infantil del cuestionario CDI. Esto es: las participantes de género femenino que presentan niveles más altos de insatisfacción corporal, tienden a puntuar más alto en los niveles de Disforia $[r=$ .505, $p<0.05]$ y de Depresión Total $[r=.491, p<0.05]$. Este resultado aparecía ya cuando se analizaban las correlaciones en la muestra general, pero el valor de estas aumenta de forma importante en el caso de las chicas: la relación entre estar insatisfechas y sentirse deprimidas parece ser más potente en este subgrupo que en la muestra general.

\section{DISCUSIÓN DE RESULTADOS}

Los resultados obtenidos en este estudio confirman que, tal y como se observa en estudios previos, los preadolescentes han comenzado ya en cierta forma a presentar niveles considerables de insatisfacción corporal. Además, al menos en la muestra escogida, las participantes de género femenino presentan mayores puntuaciones de insatisfacción corporal que los participantes de género masculino. Sin embargo, no parece haber un aumento significativo de esta insatisfacción entre un curso y otro [ $5^{\circ}$ y $6^{\circ}$ ] tanto para el género masculino como para el femenino.

Por otro lado, los participantes que tienen mayor insatisfacción corporal tienden a presentar algún tipo de puntuación relacionada con niveles más bajos de autoestima, especialmente las chicas mayores, quienes obtienen puntuaciones mayores en Disforia que los chicos y que las chicas más pequeñas.

Podría decirse que los resultados obtenidos en el presente estudio reflejan solamente una pequeña parte de la conciencia corporal y comportamientos relacionados con la insatisfacción corporal que sufren actualmente los preadolescentes. Sin embargo, los resultados aquí obtenidos coinciden de manera más o menos significativa en diferentes aspectos con otros estudios que tratan los mismos temas o similares, como es el realizado por De Gracia, Marcó y trujano [2007], también el de Moreno y Ortiz [2009], Chávez [2007], Ruiz et al. [2004], o Trujano et al. [2010], entre otros.

\section{CONCLUSIONES GENERALES Y LÍNEAS DE CARA AL FUTURO}

Tal y como hemos visto a lo largo del presente estudio, la adolescencia es una etapa del crecimiento esencial para la adquisición de hábitos y de comportamientos con respecto a la salud, pues muchos factores determinantes de diversos trastornos o enfermedades empiezan en esta etapa, en la que parece que el culto por la imagen corporal tiene mayor importancia, en este caso adentrándonos en una sociedad o cultura donde la delgadez y el atractivo físico están intimamente relacionados, especialmente para las chicas. Es por ello por lo que los adolescentes se comparan constantemente con la apariencia de los ideales sociales que actúan como modelos en esta cultura, la mayoría de los cuales son poco realistas y pueden llegar a generar altos niveles de insatisfacción corporal, lo cual puede derivar en posibles TCA o estados depresivos, ansiedad, baja autoestima, etc. 
Aun teniendo en cuenta las limitaciones de este pequeño estudio, se ha podido confirmar que, aunque no en un grado muy elevado, sí aparecen ya en estas edades ligeros niveles de insatisfacción corporal entre los chicos y las chicas, y que estos niveles se relacionan también con la autoestima que presentan, al menos en parte. Además, podemos también afirmar respecto a la hipótesis inicial que las mujeres tienden a sentirse más insatisfechas con su cuerpo que los hombres, insatisfacción que además aumenta con la edad, es decir, las chicas mayores en edades correspondientes a la pubertad tendrían niveles más elevados de insatisfacción corporal que las chicas más jóvenes.

Con todo ello resulta claro que, ante los TCA, se precisa una adecuada orientación psicopedagógica necesaria desde la más temprana edad, contando con la participación activa del entorno familiar y el contexto educativo. Conviene, de esta manera, abordar temas como la higiene mental, el desarrollo de la personalidad, la educación para la salud, y otros aspectos educativos y escolares relevantes que puedan ser factores influyentes en el desarrollo de un TCA.

Podemos concluir, por tanto, que la construcción de la imagen corporal debería cuidarse, ya desde la preadolescencia, atendiendo a los fenómenos socioculturales que influyen en las preocupaciones de los adolescentes y que afectan de manera negativa a su salud. Por ello, es imprescindible llevar a cabo programas de carácter preventivo de trastornos alimentarios ya en edades anteriores, con el fin de impedir el surgimiento o incremento del problema.

Si bien los resultados de esta investigación no pueden generalizarse a toda la población de niños/ as de estas edades, debido a las limitaciones de la misma (tamaño de la muestra, etnia de los participantes, nivel socio-económico y cultural..., podrían ser una pista más para reconocer que los grupos estudiantiles son una población delicada de alto riesgo para desarrollar posibles TCA, trastornos que están muy relacionados con la preocupación por la imagen corporal y con la baja autoestima. Todos estos elementos deben ser clave para el diseño de futuras investigaciones y programas preventivos que son, sin duda, necesarios. 


\section{REFERENCIAS BIBLIOGRÁFICAS}

Brunch, H. [1962). Perceptual and conceptual disturbances in anorexia nerviosa. Psychosomatic Medicine, 24, 187-194.

Chávez, H. A. E. [2007]. Actitudes y conductasalimentariasenadolescentes y surelación con indice de masa corporal. Saludcomunitaria. Universidad Autónoma de Nuevo León, Facultad de Enfermería. Red de enfermería de América Latina.

Collins, M. E. [1991]. Body figure perceptions and preferences among preadolescent children. International Journal of Eating Disorders, 10, 199-208.

De Gracia, M., Marcó, M. y Trujano, P. [2007]. Factores asociados a la conducta alimentaria en preadolescentes. Psicothema, [19] 4, 646-653.

Espina, A., Ortego, M. A., Ochoa de Alda, I., Yenes, F., \&Alemán, A. [2001]. La imagen corporal en los trastornos alimentarios. Psicothema, 13 [4], 533-538.

Gallego del Castillo F. [2010]. Esquema corporal y praxia: bases conceptuales. España: Wanceulen Editorial Deportiva.

Gardner, R. M. [1996]. Methodo logicalissues in assessment of the perceptual component of body image disturbance. British Journal of Psychology, 87, 327-337.

Gómez, P.M. [2013].Insatisfacción con la imagen corporal y malestar-emocional: un estudio de mediación múltiple. Trabajo de fin de Master. Master Universitario de Estudios Avanzados en Educación Primaria. Facultad de Educación de la Universidad Complutense de Madrid. Madrid. Recuperado de: http://eprints.ucm.es/22650/1/TESINA.pdf

Kovacs, M. [1992]. CDI. Inventario de Depresión Infantil. Madrid: TEA Ediciones[Adapt. Del Barrio, V. \& Carrasco, M. A., 2004].

Le Boulch, J. [1986]. La educación por el movimiento. Barcelona: Paidós Ibérica.

Limiñana Gras, R. M. [2013]. Imagen corporal, identidad de género y alimentación. Dossiers Feministes, 17, 99-104.

Moreno, M. A. \& Ortiz, G. R. [2009]. Trastorno Alimentario y su Relación con la Imagen Corporal y la Autoestima en Adolescentes. Terapia Psicológica, 27 [2], 181-190.

Pastor, R. \& Bonilla, A. [2000]. Identidades y cuerpo: El efecto de las normas genéricas. Papeles del Psicólogo, 75.

Portela de Santana, M. L., Da Costa, H., Mora M. \&Raich, R. M. [2012]. La epidemiología y los factores de riesgo de los trastornos alimentarios en la adolescencia; una revisión. Nutrición Hospitalaria, 27[2], 391-401.

Raich, R.M. [2010]. Imagen corporal. Conocer y valorar el propio cuerpo. Madrid: Pirámide D.L.

Rosen, J.C. [1992]. Body image disorder: Definition, development, and contribution to eating 
disorders. In J.H. Crowther, D.L. Tennenbaum, S.E. Hobfoll, \& M.A.P. Stephens [eds.], The aetiology of bulimia: The individual and familial context [157-177). Washington D.C.: Hemisphere Publishing Corporation.

Ruíz, M. A. O., Vázquez, A. R., Mateo, G. C, Galdós, C. J., Álvarez, R. G. \& Mancilla, D. J. M. [2004] Presencia de trastornos alimentarios y su sintomatología en relación con influencias culturales e insatisfacción corporal entre estudiantes hombres y mujeres. Revista Psicología, 1 (1).

Salaberria, K., Rodríguez, S. \& Cruz, S. [2007]. Percepción de la imagen corporal. Cuadernos de Ciencias Médicas, 8, 171- 183.

Schilder, P. (1978). The image and appearance of the human body. New York: International Universities Press.

Trujano, P., Nava,P., De Gracia, M., Limón, G., Alatriste, A. L. \& Merino M. T. [2010]. Trastorno de la imagen corporal: Un estudio con preadolescentes y reflexiones desde la perspectiva de género. Anales de Psicología, 26 [2], 279-287. 